\title{
NEW VALIDATION OF SI CROSS-SECTION USING SILICA SAND
}

\author{
Tomáš Czakoj ${ }^{1}$, Michal Košt’ál ${ }^{1}$, Evžen Losa $^{1}$ and Vojtěch Rypar ${ }^{1}$ \\ ${ }^{1}$ Research Centre $\check{R} e \check{z}$ \\ Hlavní 130, Husinec Řež. Czech Republic \\ tomas.czakoj@cvrez.cz,michal.kostal@cvrez.cz, evzen.losa@cvrez.cz,vojtech.rypar@cvrez.cz
}

\begin{abstract}
Silicon cross-sections, being important for a criticality safety of final spent fuel disposals, were recently reevaluated within the IAEA INDEN project. Similarly, the thermal Scattering Law matrix for silicon dioxide, which is also important for criticality safety, was also reevaluated in the ENDF/B-VIII.0 library. Due to these reasons, a series of validation experiments with silica sand were performed at the LR-0 reactor. This paper describes these validation experiments, which used two different amounts of silica sand placed in the core. The first part of validation was carried out as critical experiments in order to benefit from the suitability of integral experiments for validation. The second part - fast neutron spectrum measurement in the sand - was performed in order to obtain knowledge of its characteristics and its agreement with a calculation. The results showed significant improvement of the Thermal Scattering Law matrix for silicon dioxide available in the ENDF/B-VIII.0 library. They also showed that the new INDEN evaluation of silicon cross-sections, together with its description in the ENDF/B-VIII.0 gives disagreement rate closest to the experiments carried out without silica sand insertions. The spectrum measurement showed that the calculations of fast neutron spectra in the sand show only slight differences between different evaluations of silicon cross-sections. However, the fast neutron spectrum is not dependent on the Thermal Scattering Law. The calculated spectra show relatively good agreement with the measurement.
\end{abstract}

KEYWORDS: INDEN evaluation, LR-0, Si capture, Neutron spectrum, Sand

\section{INTRODUCTION}

Despite the fact that the silicon is not substantially present in the present reactors, it can be assumed as important nuclide from the point of view of criticality safety. Silicon, as a part of rocks, will be nonnegligible nuclide in the final spent fuel disposals. Moreover, silicon is nowadays part of concrete, which is used in spent fuel casks. Last but not least, silicon is irradiated in the reactor (silicon doping), or it is considered as material for fuel cladding (in the form of silicon carbide). Besides these facts, there are repeated discussions about the use of nuclear reactors in space. Even in this case, it is necessary to take into account criticality safety, and one postulated accident is the burial of the reactor in the sand [1]. Due to these reasons, silicon and its compounds are important materials for the nuclear industry, which means that it is important to know its properties properly.

The elastic scattering and the radiative capture cross-sections of stable silicon isotopes were not reevaluated in the last release of ENDF/B-VIII.0 library [2,3]. This version contains data adopted from the previous releases of this series [3]. Nevertheless, the silicon cross-sections were reevaluated under the 
INDEN project by a collaboration of Oak Ridge National Laboratory and IAEA [3]. These data are not available in the present major versions of nuclear data libraries.

Another important fact is the update of the Thermal Scattering sub-library of ENDF/B-VIII.0. Among others, this version includes an updated silicon dioxide Thermal Scattering Law matrix (hereinafter TSL) and newly added TSL for silicon carbide [2].

These new facts mean that it is very important to validate these data precisely. Nowadays, there are several benchmarks containing a sufficient amount of silicon. The most suitable is benchmark describing the experiment performed at the Big Physical Stand in Russia. This experiment contained silicon in the form of silicon dioxide sand. The experiment was conducted using two types of fuel - based on plutonium or uranium [4]. This benchmark was used in the preparation of the INDEN reevaluation of silicon [3].

It is important to validate the new data in an experiment which was not used in the preparation of new nuclear data. Due to this reason, the new series of integral experiments were performed at the LR-0 reactor. These experiments, as well as the Russian experiment, contained silicon in the form of silica sand.

\section{EXPERIMENTS AND CALCULATIONS}

The experiments were conducted at the LR-0 reactor, located at Řež, Czech Republic. The reactor is a versatile light-water, zero-power, pool-type reactor. It is used for measurement of neutron-physical characteristics of the VVER type reactors, studies of neutronic properties of inserted materials, and measurement of spectral averaged cross-sections. The reactor is operated with a various distribution of fuel assemblies, the properties of which are similar to the fuel of VVER power plants. The reactivity of the reactor can be controlled by a change in the moderator level or by the control clusters. The reactivity of all experiments described in this paper was controlled by the change in the moderator level, and fuel was only partially flooded by water.

Typical thermal neutron flux (below $1 \mathrm{eV}$ ) during activation experiments of the smaller core is about $3 \times 10^{7} \mathrm{~cm}^{-2} \mathrm{~s}^{-1}$ and a fast flux (above $100 \mathrm{keV}$ ) is about $6 \times 10^{7} \mathrm{~cm}^{-2} \mathrm{~s}^{-1}$. The larger core has thermal neutron flux about $9 \times 10^{6} \mathrm{~cm}^{-2} \mathrm{~s}^{-1}$, fast flux about $2 \times 10^{7} \mathrm{~cm}^{-2} \mathrm{~s}^{-1}$. Neutron flux in other types of experiments (pin power density measurements, critical experiments, etc.) is several orders lower.

\subsection{Experiments - Criticality}

Experiments were performed with a different amount of sand. The first experiment consisted of one hexagonal aluminum module partially filled with silica sand. Totally, almost $50 \mathrm{~kg}$ of sand was used, and an average density was $1.439 \mathrm{~g} / \mathrm{cm}^{3}$. A height of sand has reached $78.2 \mathrm{~cm}$, so the height of sand was approximately two-thirds of the fission column. The module with sand was closely surrounded by six fuel assemblies with an average enrichment of $3.3 \%$ (see the left side of Figure 1).

The second experiment consisted of seven modules filled with sand. The modules had the same properties as the module in the previous case. Totally, $350 \mathrm{~kg}$ of silica sand was used, and the averaged density of sand was $1.453 \mathrm{~g} / \mathrm{cm}^{3}$. The heights of sand were between 74.5 and $78.1 \mathrm{~cm}$. The modules with sand were placed in a hexagonal lattice, and they were tightly surrounded by twelve fuel assemblies with an average enrichment of $3.6 \%$ (see the right side of Figure 1). The $3.6 \%$ enrichment was used due to the insufficient number of $3.3 \%$ fuel assemblies.

The experiments were also performed without insertion, on that account, only the aluminum module was inserted into the core. These types of experiments were used to determine the influence of sand on criticality. All experiments were repeated several times, and the average heights of the moderator are listed in Table II. 


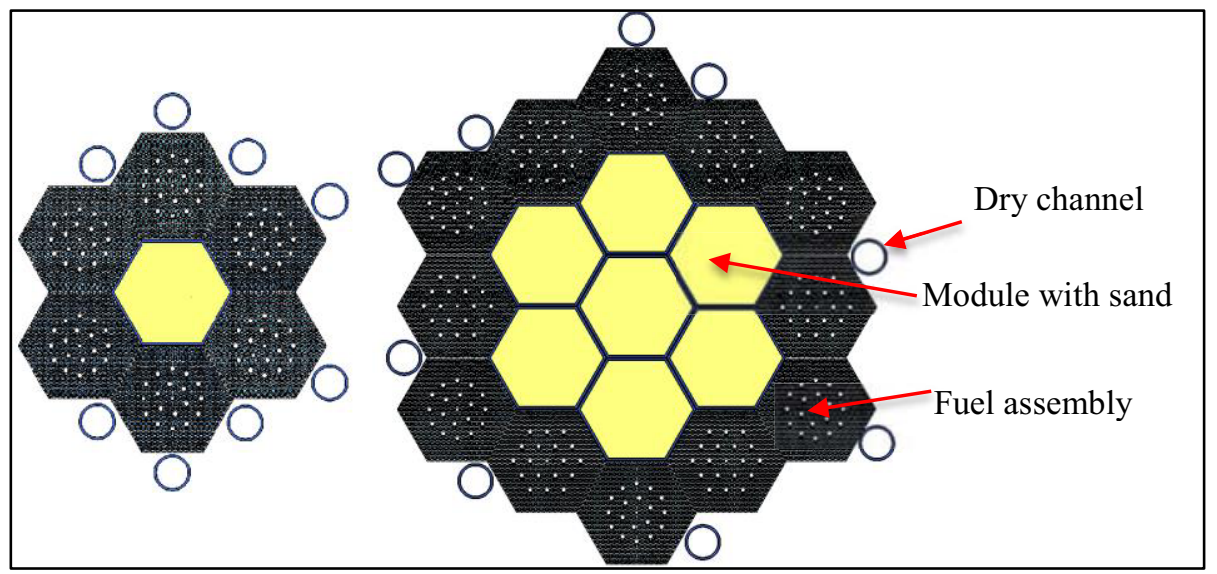

Figure 1. Schematic drawing of experiments with modules with sand.

\subsection{Experiments - Neutron Spectrometry}

Measurement of the neutron spectrum was carried out for the case with seven channels with sand. An aluminum cylindrical channel was placed into the center of the central module with sand. This cylindrical channel was not filled with sand, and the channel served as a guide tube for the spectrometer (see Figure 2). Due to the presence of the cylindrical channel, the height of sand in the central channel has increased by $11 \mathrm{~cm}$ (the mass of sand was conserved).

The high-energy region of the neutron spectrum was measured by the method of recoiled protons in the energy range $1.2-10 \mathrm{MeV}$ with $0.1 \mathrm{MeV}$-wide energy groups. The measurements were taken with a stilbene scintillator detector. The stilbene scintillator crystal sized $1 \times 1 \mathrm{~cm}$ with neutron and gamma pulse shape discrimination was used (hereinafter Stilbene), more information about the used measurement apparatus can be found in [5], calibration of the detector is well-described in [6]. The thermal energy region measurement was out of the scope of the paper, and it was measured later using reaction rate measurement.

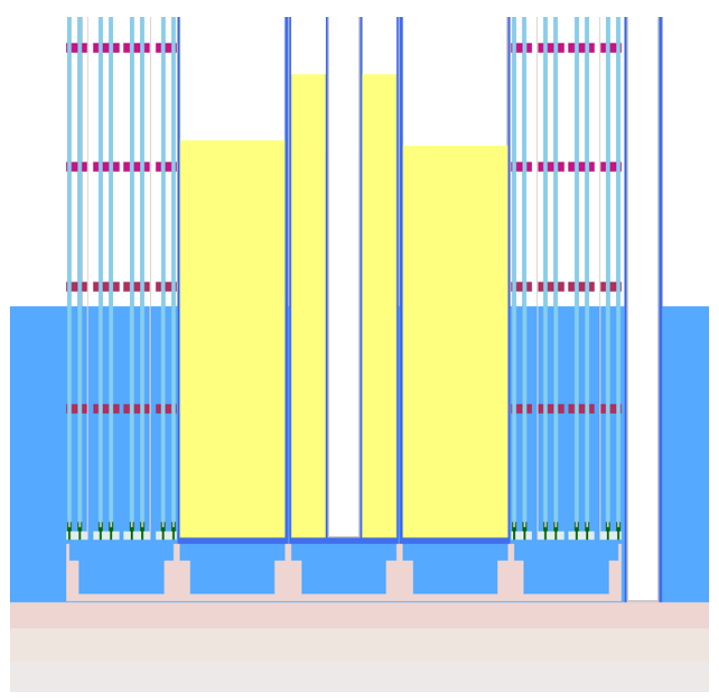

Figure 2. Illustration of central tube for neutron spectrometry. 


\subsection{Calculations}

Calculations were made using the Monte Carlo method employing the MCNP6.2 code [7]. All materials excluding silicon in the sand and uranium were defined in the ENDF/B-VII.1 library [8]. Uranium was defined in the ENDF/B-VIII.0 library [2]. The computational models for both experiments contained silicon defined in INDEN evaluation [3], ENDF/B-VII.1 [8], and ENDF/B-VIII.0 [2]. The thermal scattering law matrix for sand was available only in ENDF/B-VII.1 and ENDF/B-VIII.0, so they were used only with the corresponding library. The TSL matrix from ENDF/B-VIII.0 was also tested with the INDEN evaluation. These calculations were also tested without application of the TSL matrix for sand.

Moreover, due to the larger influence of sand, silicon in the large sand experiment was also defined in other libraries. These libraries were as follows: JENDL-3.3 [9], JENDL-4.0 [10], JEFF-3.1 [11], JEFF-3.2 [12], JEFF-3.3 [13], CENDL3.1 [14], TENDL-2017 [15, 16], and ROSFOND-2010 [17]. The thermal scattering law matrix for sand was not available in these libraries, so no TSL library for sand was used in these calculations.

The measured critical moderator levels were used in the calculation. MCNP criticality calculations employed 40,000 source neutrons per generation and 5,000 active generations. The skipping of the first 50 generations assured correct source convergence.

Spectra calculations were performed using silicon defined in the INDEN evaluation or ENDF/B-VII.1 library. The influence of the TSL matrix on spectra was also determined. Spectra calculations were performed with 40,000 neutrons per generation and 1,000,050 generations (1,000,000 active and 50 inactive). This setting assured statistical uncertainty below $2 \%$ in the energy below $7.5 \mathrm{MeV}$ and statistical uncertainty below $5 \%$ in the range of $7.5-10 \mathrm{MeV}$. The obtained results were modified by Gaussian broadening using parameters for the stilbene detector.

The experimental uncertainties in $\mathrm{k}_{\mathrm{eff}}$ associated with the experiments are mostly caused by manufacturing and measurement tolerances. The identified sources of uncertainties are summarized in Table I. The influence of these occurrences was assessed by one-sided direct perturbations of the model. And assuming that the sources are uncorrelated, a total uncertainty was determined as the square root of the sum of squares of each source.

Table I. Sources of uncertainties in experiments.

\begin{tabular}{|c|c|c|c|}
\hline Source of uncertainty & Value $(1 \sigma)$ & $\begin{array}{c}\text { Case with smaller } \\
\text { amount of sand }[\mathrm{pcm}]\end{array}$ & $\begin{array}{c}\text { Case with higher } \\
\text { amount of sand [pcm] }\end{array}$ \\
\hline Fuel assembly pitch & $0.15[\mathrm{~cm}]$ & 94 & 30 \\
\hline Fuel Density & $0.0093[\mathrm{~g} / \mathrm{cm} 3]$ & 12 & 7 \\
\hline Fuel Enrichment & $0.01[\mathrm{wt} . \%]$ & 38 & 34 \\
\hline Fuel Impurities & $0.115[\mathrm{wt} . \%$ relative to U] & 35 & 64 \\
\hline Moderator density & $0.0004[\mathrm{~g} / \mathrm{cm} 3]$ & 10 & 47 \\
\hline Moderator impurities & $1[\mathrm{ppm} \mathrm{BE}]$ & 23 & 12 \\
\hline Moderator level & $0.058[\mathrm{~cm}]$ & 12 & 84 \\
\hline Outer cladding diameter & $0.0016[\mathrm{~cm}]$ & 47 & 1 \\
\hline Sand density & $0.0015[\mathrm{~g} / \mathrm{cm} 3]$ & 4 & 16 \\
\hline Sand impurity & $0.40[\mathrm{wt} . \%]$ & 6 & 126 \\
\hline Total & & 121 & \\
\hline
\end{tabular}




\subsection{Comparison of Calculation and Experiments - Criticality \\ 3. RESULTS AND DISCUSSION}

The experimentally determined critical configurations listed in Table II show that the insertion of the sand has a positive impact on reactivity, as the amount of moderator needed to reach criticality is lower. The calculated $\mathrm{k}_{\mathrm{eff}}$ for the small experiment without sand insertion is $0.99958 \pm 0.00005$, the calculated $\mathrm{k}_{\text {eff }}$ for the large experiment without sand is $1.00340 \pm 0.00005$. It is apparent that the large experiment has a larger bias. This bias is most likely caused by modeling simplifications and ${ }^{234} \mathrm{U}$ content, which is neglected in the benchmark model.

Table II. Critical moderator levels.

\begin{tabular}{|c|c|c|}
\hline & Case with smaller amount of sand & Case with higher amount of sand \\
\hline With insertion & $48.83 \pm 0.06 \mathrm{~cm}$ & $45.73 \pm 0.01 \mathrm{~cm}$ \\
\hline Without insertion & $55.72 \pm 0.03 \mathrm{~cm}$ & $57.47 \pm 0.01 \mathrm{~cm}$ \\
\hline
\end{tabular}

The results of the criticality calculations of the small experiment are depicted in Figure 3. It is obvious that the new TSL matrix rapidly decreases the difference between empty and filled experiment. The difference between ENDF/B-VIII.0 and INDEN cross-sections is about $40 \mathrm{pcm}$, and the difference between the calculation of empty and filled experiments is nearly $20 \mathrm{pcm}$.

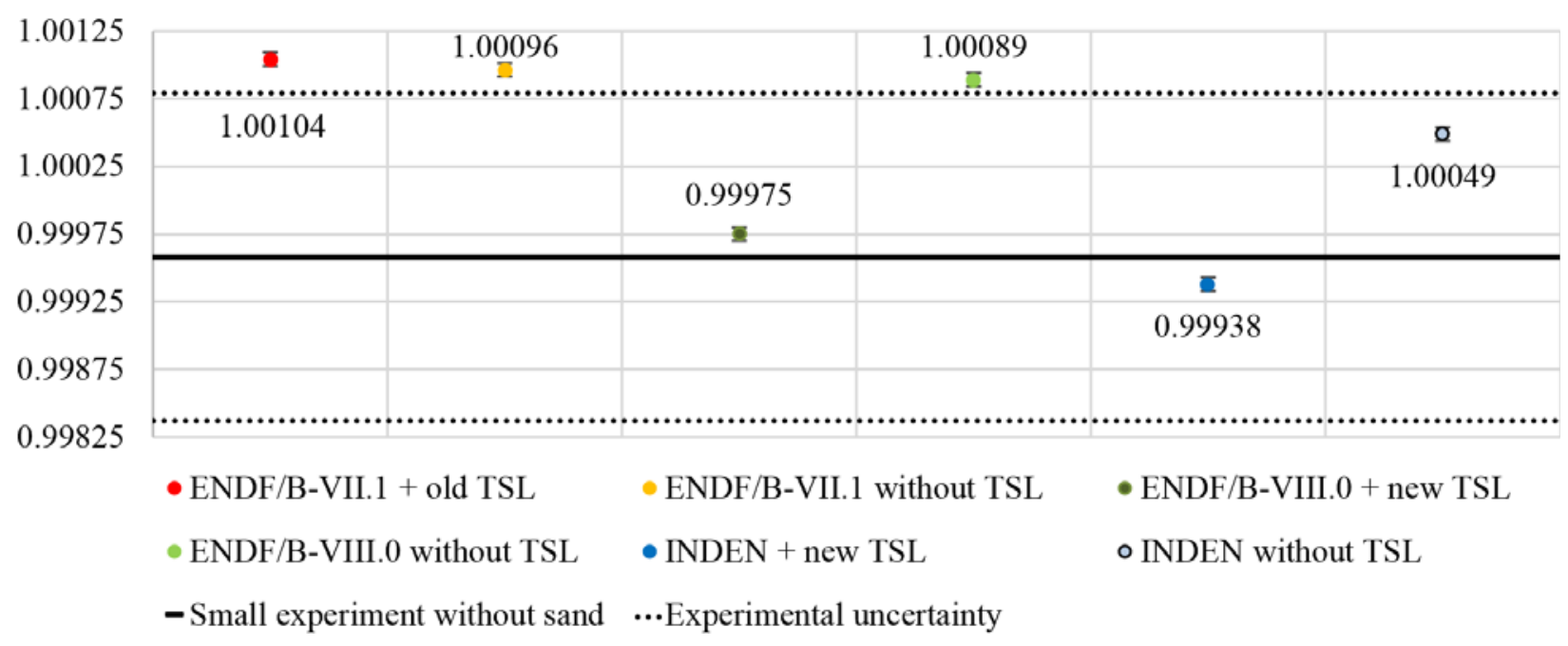

Figure 3. Multiplication factor for small experiment with silicon in different nuclear data libraries.

The results for the large experiment are depicted in Figure 4 and Figure 5. Not surprisingly, the trend in Figure 4 is the same as in Figure 3. The application of the new TSL matrix considerably decreases the difference between the calculation of the experiment with and without sand, and both ENDF/B-VIII.0 and INDEN evaluation give reasonable results. The large differences between experiments in Figure 5 are caused by the nonexistence of the TSL matrix for sand in the applied libraries for silicon in the sand. The differences between results in this figure are within $60 \mathrm{pcm}$, and TENDL-2017 gives the best results. Oppositely, CENDL3.1 gives results farthest from the experiment without sand. 


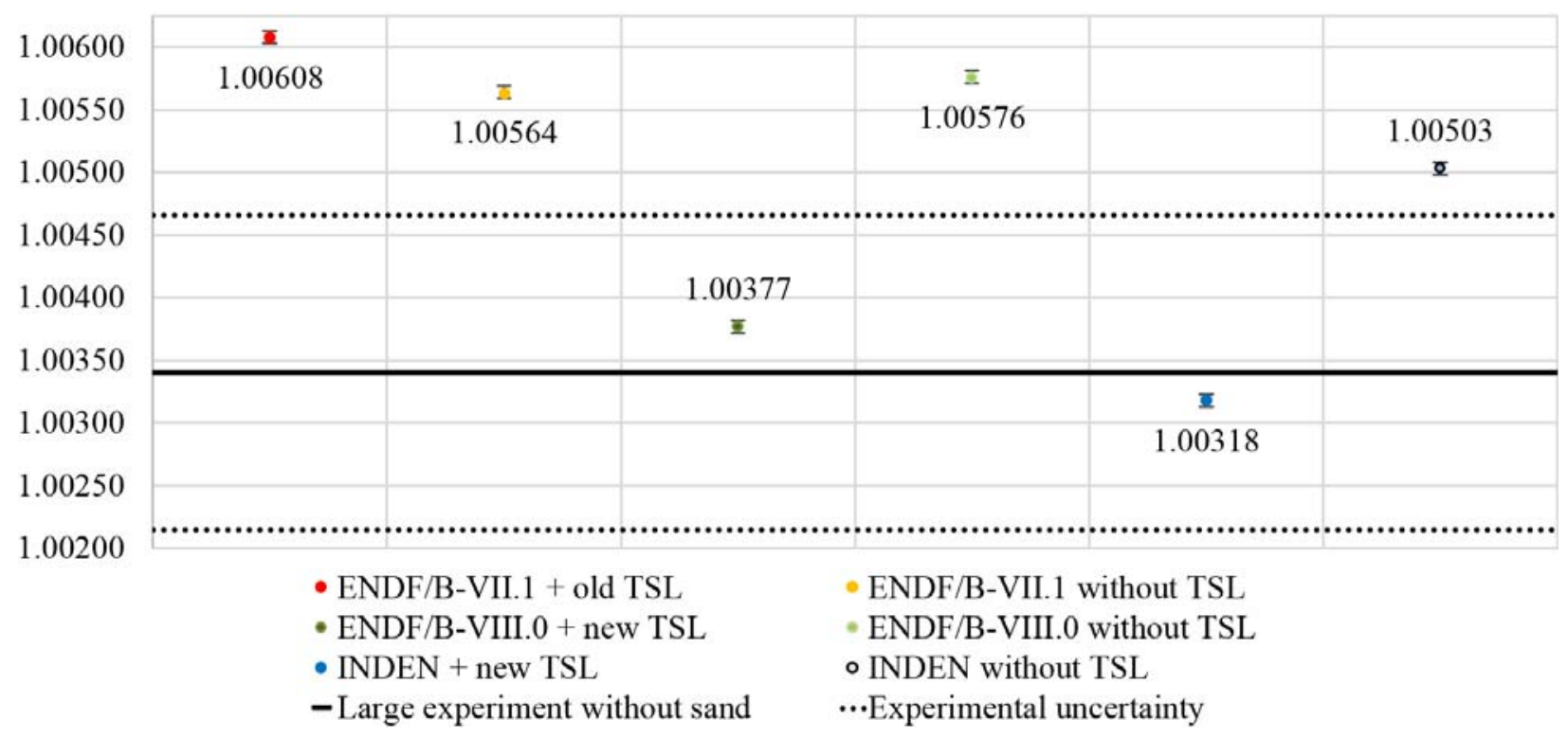

Figure 4. Multiplication factor for large experiment with silicon in different nuclear data libraries.

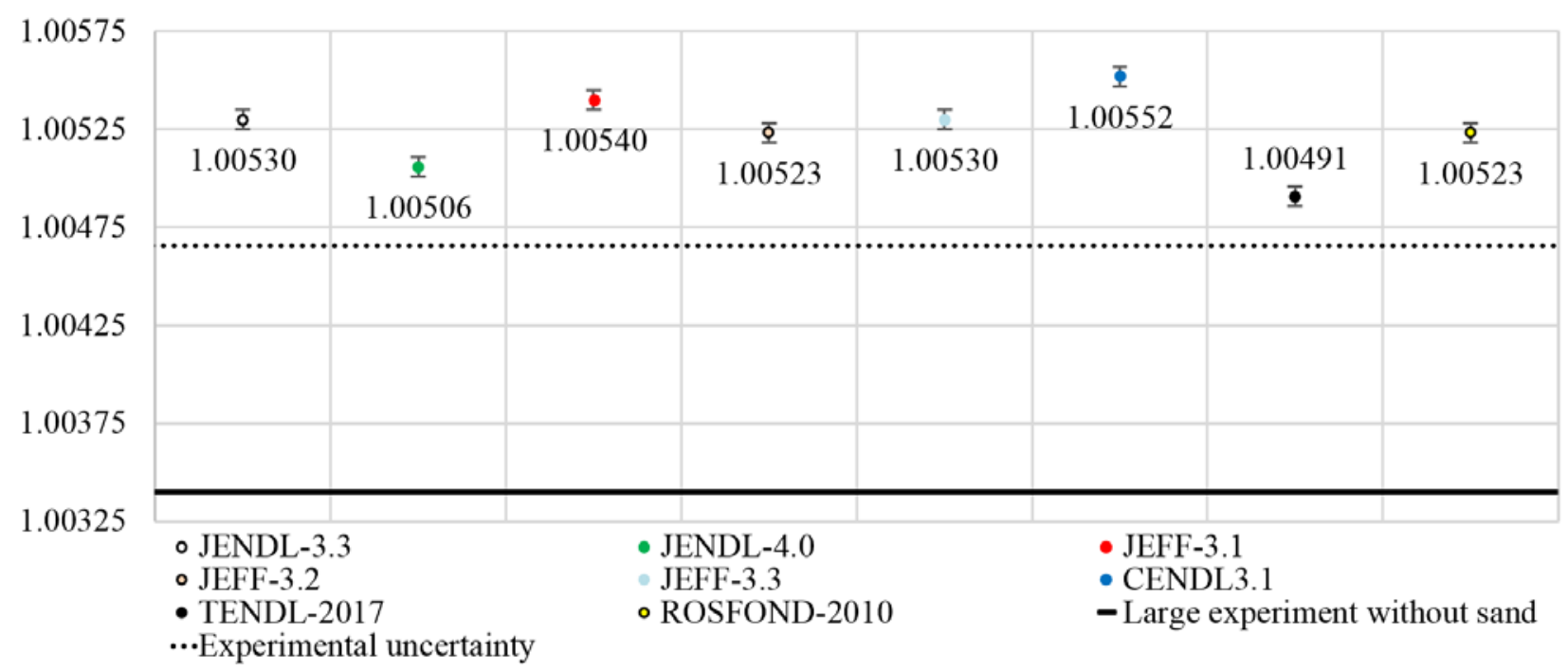

Figure 5. Multiplication factors for large experiment with silicon in other nuclear data libraries.

\subsection{Comparison of Calculation and Experiments - Neutron Spectrometry}

Figure 6 shows a comparison of the experimentally determined and calculated spectra in the sand. The figure shows only one calculated spectrum because the differences between calculated spectra were only in the thermal region, which is not covered by the measurement. The experimentally determined spectrum is normalized to 1 in integral above $3 \mathrm{MeV}$.

For the experiment, the statistical uncertainties along with the uncertainties of calibration and uncertainty in detector resolution are in the range of $5-10 \%$ below $2.5 \mathrm{MeV}$, about $5-6 \%$ in $2.5-7 \mathrm{MeV}$, and reach $10 \%$ in the energy region above $7 \mathrm{MeV}$. The higher uncertainties in the lowest energy region are caused 
by the higher influence of uncertainties of the calibration, and the higher uncertainties in the highest energy region are caused by the problem with a spectrum deconvolution.

As can be seen in Figure 6, the calculated spectra are almost the same and the differences between them are within $2.5 \%$. Due to this reason, the sand library selection has almost no influence on the calculation of the neutron stilbene detector response and only one calculated spectrum with silicon in INDEN is depicted. The spectrum measured in the sand is in relatively good agreement with calculations. The calculations significantly overpredict peak around $2.3 \mathrm{MeV}$, but this is caused by problems with the resolution of the stilbene spectrometer.

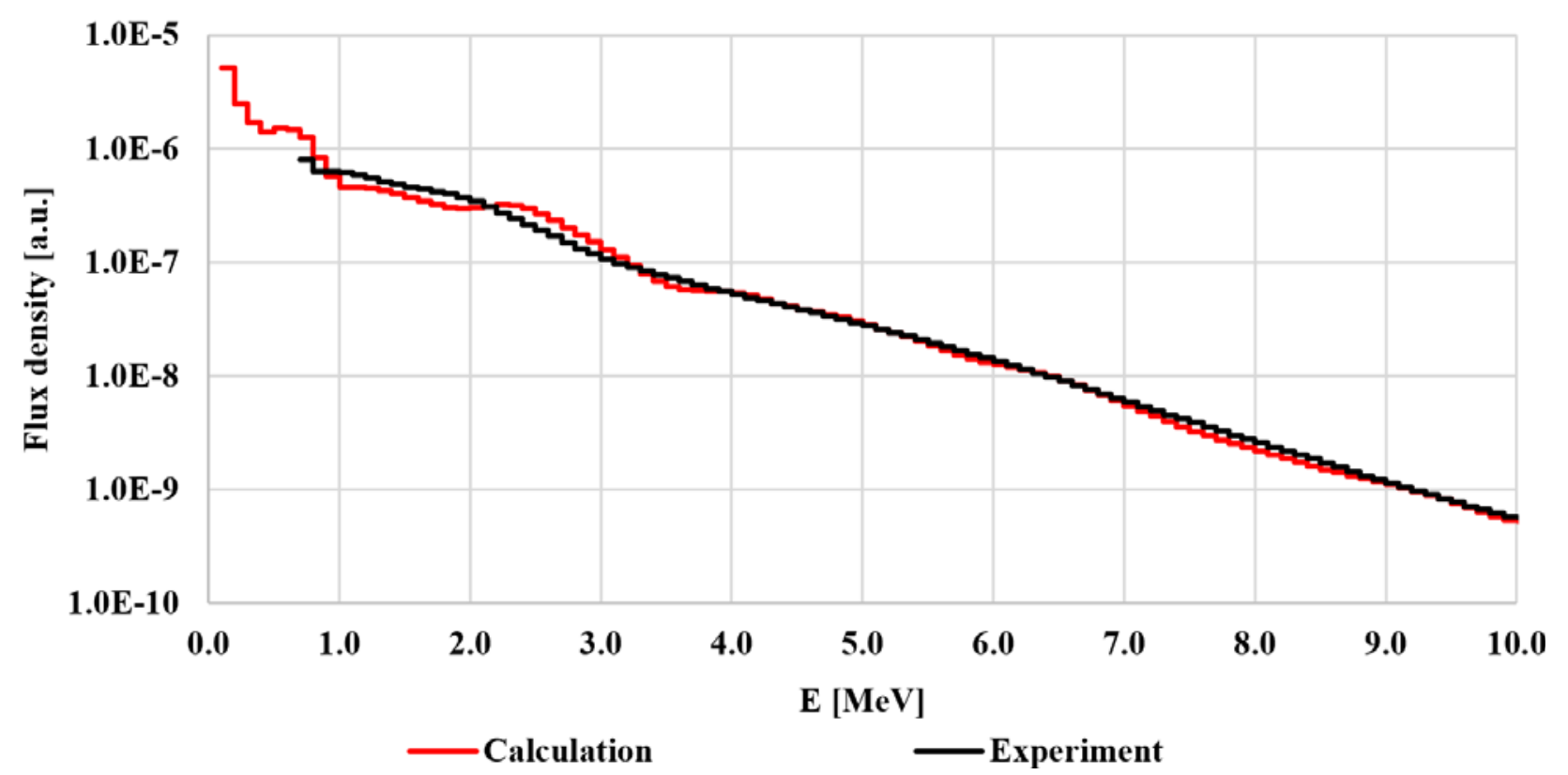

Figure 6. Neutron spectrum in sand.

\section{CONCLUSIONS}

Since the silicon cross-sections were reevaluated under the INDEN project and due to the update of the TSL matrix for sand in the ENDF/B-VIII.0 library, the set of experiments using silica sand was performed for validation of these new data. The calculations of critical experiments were performed using a wide range of nuclear data libraries. These calculations show the significant influence of the new TSL matrix for sand. Its application significantly decreases discrepancies between experiments with and without insertion. Opposite to that, the old TSL matrix for sand increases the discrepancy between experiments. The ENDF/B-VIII.0 and INDEN evaluation of silicon cross-sections, in case of application TSL matrix, give satisfactory results, the INDEN slightly underestimates the experiments, and oppositely ENDF/BVIII.0 overestimates the experiments. Comparison of calculations of spectrum in the sand with the measurement showed a large overestimation of neutron flux in energies around $2.3 \mathrm{MeV}$, other energies are in relatively good agreement.

The experiments with silica sand will be extended at least by the reaction rates measurement. This experiment will describe also the thermal and epithermal part of neutron spectra. A benchmark describing the critical experiments is in preparation, and a benchmark describing the future reaction rates measurement is also planned. 


\section{ACKNOWLEDGMENTS}

Presented results were obtained with the use of the infrastructure Reactors LVR-15 and LR-0, which is financially supported by the Ministry of Education, Youth and Sports - project LM2015074 and also financially supported by the Ministry of Education, Youth and Sports Czech Republic - project LQ1603 Research for SUSEN. This work has been realized within the SUSEN Project (established in the framework of the European Regional Development Fund (ERDF) in project CZ.1.05/2.1.00/03.0108 and of the European Structural Funds and Investment Funds (ESIF) in the project CZ.02.1.01/0.0/0.0/15_008/0000293), which is financially supported by the Ministry of Education, Youth and Sports - project LM2015093 Infrastructure SUSEN.

\section{REFERENCES}

1. S. Bragg-Sitton, J. Bess, J. Werner, G. Harms, S. Bailey "Reactor Testing and Qualification: Prioritized High-level Criticality Testing Needs"

2. Brown DA, Chadwick MB, Capote R, et al "ENDF/B-VIII.0: The 8th Major Release of the Nuclear Reaction Data Library with CIELO-project Cross Sections, New Standards and Thermal Scattering Data". Nucl Data Sheets 148, pp. 1-142 (2018)

3. Pigni MT, Guber KH, Arbanas G, et al "Evaluation and Validation of 28,29,30SI Cross Sections in the Resolved Resonance Region". Oak Ridge, United States

4. Gagarinski AY (Russian RC "Kurchatov I) "Russian nuclear criticality experiments. Status and prospects". In: Proceedings of the International Conference Nuclear Energy for New Europe 2003. Nuclear Society of Slovenia, Slovenia, p 10

5. Veškrna M, Matěj Z, Mravec F, et al "Digitalized two parametric system for gamma/neutron spectrometry". In: 18th Topical Meeting of the Radiation Protection and Shielding Division of ANS, Knoxville, TN, Sept. pp 14-18

6. Koštál M, Šoltés J, Viererbl L, et al "Measurement of neutron spectra in a silicon filtered neutron beam using stilbene detectors at the LVR-15 research reactor". Appl Radiat Isot 128, pp. 41-48 (2017)

7. $\quad$ Werner CJ "MCNP Users Manual-Code Version 6.2"

8. Chadwick MB, Herman M, Obložinský P, et al "ENDF/B-VII.1 Nuclear Data for Science and Technology: Cross Sections, Covariances, Fission Product Yields and Decay Data". Nucl Data Sheets 112, pp. 2887-2996 (2011)

9. Shibata K, Kawano T, Nakagawa T, et al "Japanese Evaluated Nuclear Data Library Version 3 Revision-3: JENDL-3.3". J Nucl Sci Technol 39, pp. 1125-1136 (2002)

10. Shibata K, Iwamoto O, Nakagawa T, et al "JENDL-4.0: A New Library for Nuclear Science and Engineering". J Nucl Sci Technol 48, pp. 1-30 (2011)

11. Santamarina A, Bernard D, Blaise P, et al The JEFF-3.1.1 Nuclear Data Library Validation Results from JEF-2.2 to JEFF-3.1.1 NEA OECD

12. OECD/NEA Data Bank "JEFF-3.2 evaluated data library - Neutron data". http://www.oecdnea.org/dbforms/data/eva/evatapes/jeff_32/

13. NEA Nuclear Data Services "JEFF-3.3". https://www.oecd-nea.org/dbdata/jeff/jeff33/index.html

14. Ge ZG, Zhao ZX, Xia HH, et al "The updated version of Chinese evaluated nuclear data library (CENDL-3.1)". J Korean Phys Soc 59, pp. 1052-1056 (2011)

15. Koning AJ, Rochman D "Modern Nuclear Data Evaluation with the TALYS Code System". Nucl Data Sheets 113, pp. 2841-2934 (2012)

16. Rochman D, Koning AJ, Sublet JC, et al "The TENDL library: Hope, reality and future". EPJ Web Conf 146, pp. 02006 (2017)

17. Zabrodskaya S V., Ignatyuk A V., Koshcheev VN, et al "ROSFOND--Russian National Library of Evaluated Neutron Data". Vopr At Nauk Tekhn Ser Yad Konst pp. 3-21 (2007) 